\title{
Why is it necessary to examine retina when the patient suffers from aplastic anemia?
}

\author{
Tomcikova $\mathrm{D}^{1}$, Gerinec $\mathrm{A}^{1}$, Busanyova $\mathrm{B}^{1}$, Gresikova $\mathrm{M}^{2}$, Biskup $\mathrm{S}^{3}$, Hortnagel $\mathrm{K}^{3}$ \\ Pediatric Ophthalmology Department, Children's University Hospital, Bratislava, Slovakia. \\ dtomcikova@gmail.com
}

\begin{abstract}
PURPOSES: To inform about a case of Revesz syndrome (RS) with initial ophthalmological symptomatology of severe proliferative vitreoretinopathy of the left eye (LE). After the aplastic anemia had developed, RS was established. The exudative retinopathy was successfully treated with photocoagulation on the right eye (RE). BACKGROUND: RS is characterized by fatal bone marrow failure, exudative retinopathy, neuroradiographic abnormalities, neurodevelopmental delay and skin abnormalities. Non-treated exudative retinopathy leads to blindness. METHODS: We report ophthalmological findings as follows: fundus photography and fluorescein angiography (FA) acquired by examinations under general anesthesia in patient with RS. Results of genetic tests helped to establish the diagnosis.

RESULTS: Two-year old Caucasian male was examined due to total retinal detachment on LE and signs of chorioretinal scarring on RE. In preoperative screening, thrombocytopenia was detected; later, severe pancytopenia developed. Considering the hematological findings and clinical appearance, we suspected RS, which was confirmed by genetic tests. We found a pathogenic mutation in gene TINF2 (variant c.865C>T;p.Pro289Ser) in a mosaic state with autosomal dominant mode of inheritance. This mutation has not been described in RS yet. Blind LE was enucleated because of dolorous neovascular glaucoma. FA of RE shows excessive areas of capillary nonperfusion with vascular abnormalities and exudation. After the photocoagulation, the visual acuity (VA) on RE remains 0.9 at the age of 7 years.

CONCLUSIONS: RS is an extremely rare condition. The initial symptomatology could be ophthalmological or hematological. The positive finding of TINF2 gene mutation helped in establishing the correct diagnosis. The ischemic retinopathy was successfully treated by photocoagulation (Fig. 6, Ref. 6). Text in PDF www.elis.sk. KEY WORDS: Revesz syndrome, aplastic anemia, exudative retinopathy, peripheral retinal ischemia, photocoagulation.
\end{abstract}

\section{Introduction}

Revesz syndrome is a telomeropathy, a rare subtype of dyskeratosis congenita (DC). It is characterized by bone marrow failure, exudative retinopathy, neuroradiographic abnormalities (namely, brain calcification), neurodevelopmental delay and integumentary abnormalities. Bone marrow failure is usually fatal. This genetically caused disorder was first described in 1992. The mostly observed gene mutation is known as TINF2 (1).

\section{Materials and methods}

In December 2012 a 2-year old Caucasian male was examined with a suspicion of intraocular tumor of LE. We detected

${ }^{1}$ Pediatric Ophthalmology Department, Children's University Hospital, Bratislava, Slovakia, ${ }^{2}$ Department of laboratory medicine, Children's University Hospital, Bratislava, Slovakia, and ${ }^{3}$ Praxis for Human Genetics, Tuebingen, Germany

Address for correspondence: D. Tomcikova, MD, Podhaj 21, SK-841 03 Bratislava, Slovakia.

Phone: +421.905463981 total retinal detachment on LE and signs of inactive chorioretinitis on RE. The pars plana vitrectomy was planned on LE. In preoperative screening, thrombocytopenia was diagnosed; later, severe pancytopenia developed as a consequence of bone marrow fail-

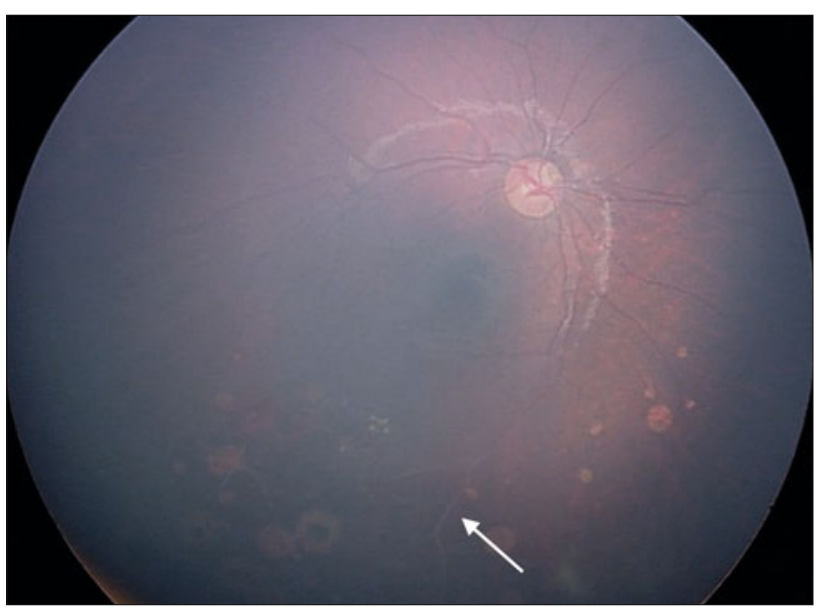

Fig. 1. Fundus of RE: areas of capillary nonperfusion, retinal vessel obliteration (arrow), round areas of chorioretinal atrophy. 

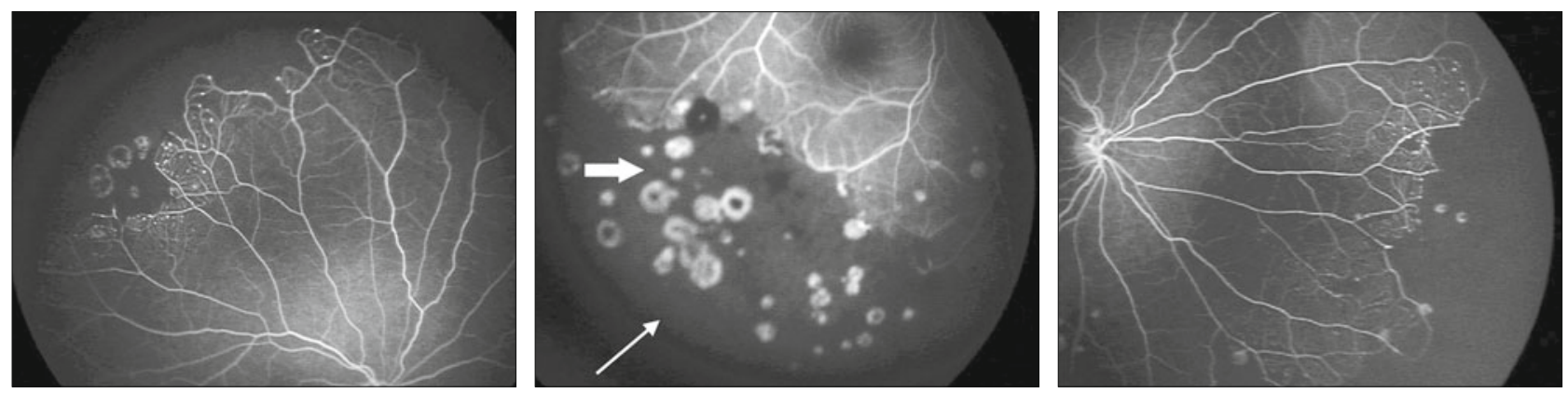

Fig. 2. FA of RE: excessive areas of capillary nonperfusion circular on the retinal periphery (arrow $\rightarrow$ ) abnormal vasculature, retinal vessel obliteration, round areas of chorioretinal atrophy (arrow $\Rightarrow$ ).

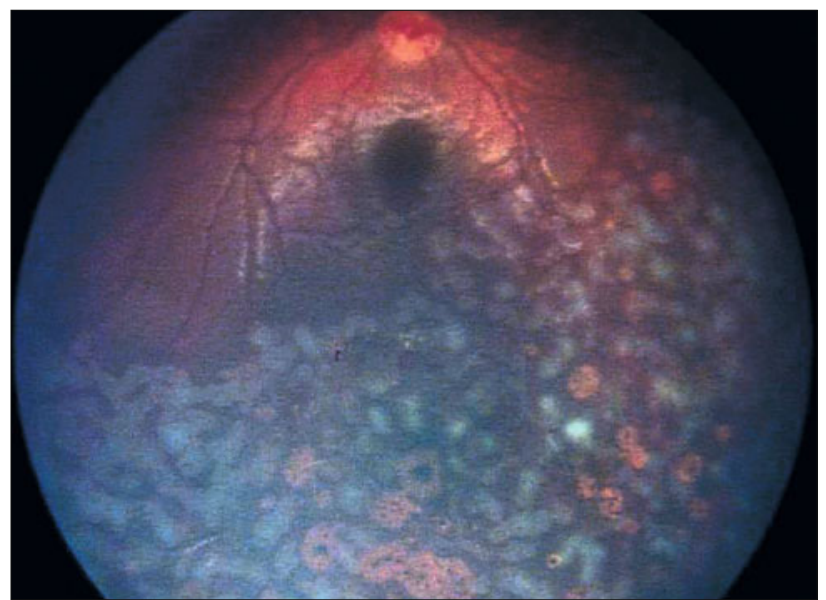

Fig. 3. Fundus of RE after photocoagulation in February 2013.

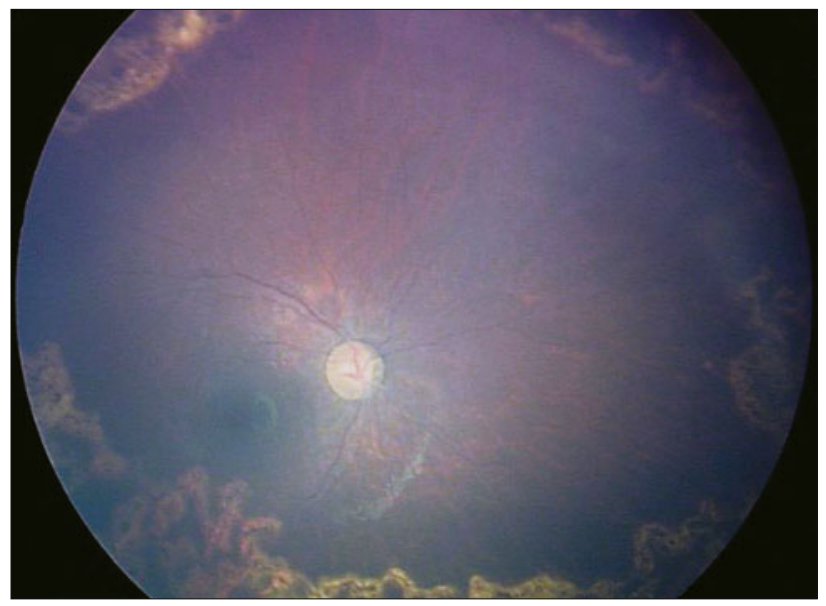

Fig. 4. Fundus of RE during control examination in February 2017.

ure. His family history was negative. Physical findings showed dystrophic nails, subtle hyperpigmentation on the neck, inguinal and scrotal areas, neurodevelopmental delay and brain calcification. Ophthalmological evaluation included fundus photography and FA, which were obtained by examinations under general anesthesia. The genetic examination was performed by Praxis für Humangenetik, Tübingen.

\section{Results}

Considering the hematological findings and clinical appearance we suspected Revesz syndrome, which was confirmed by molecular genetic tests. The mutation in gene TINF2 (variant c.865C>T;p.Pro289Ser) was found in a mosaic state with autosomal dominant mode of inheritance. Left eye of the patient was enucleated because of dolorous neovascular glaucoma after unsuccessful vitrectomy in January 2014. FA of the right eye showed excessive areas of capillary nonperfusion circular on the retinal periphery, abnormal vasculature, retinal vessel obliteration and exudation mostly on borders between vascular and avascular retina. Round areas of chorioretinal atrophy were also found (Figs 1 and 2). After the photocoagulation had been performed (Fig. 3), the visual acuity (VA) on RE remains 0.9 , at the age of 7 years. Due to the slowly progressing disease and development of new avascular areas, the consecutive photocoagulation was performed in August 2017 (Figs 4, 5 and 6).

\section{Discussion}

Revesz syndrome is a rare subtype of dyskeratosis congenita (DC), in which exudative retinopathy is found more often than in other types of DC. Detailed ophthalmological findings have rarely been publishes. In our patient, we found a mutation in gene TINF2 (variant c.865C>T;p.Pro289Ser) in a mosaic state in conjunction with severe exudative retinopathy in both eyes. The right eye was successfully treated with photocoagulation. Sharma et al (1) referred about siblings with signs of familiar exudative vitreoretinopathy (FEVR), which was followed by bone marrow failure and restrictive pulmonary disease. Genetic evaluation shows a mutation in TERT gene. This led to the diagnosis of DC with ophthalmologic phenotype of exudative retinopathy. In DC, 11 genes are known (ACD, CTC1, DKC1, NHP2, NOP10, PARN, RTEL1, TERC, TERT, TINF2, WRAP53) All of them cause different variations of DC (4). The inheritance of DC can also differ. It can be X-linked recessive, autosomal dominant, or autosomal recessive depending on the molecular defect (1). This heterogeneity may cause a phenotypic variety of clinical findings in DC, and variety in expression of exudative retinopathy and other pediatric retinal vasculopaties, including Coats' disease (2, 3). NDP gene 


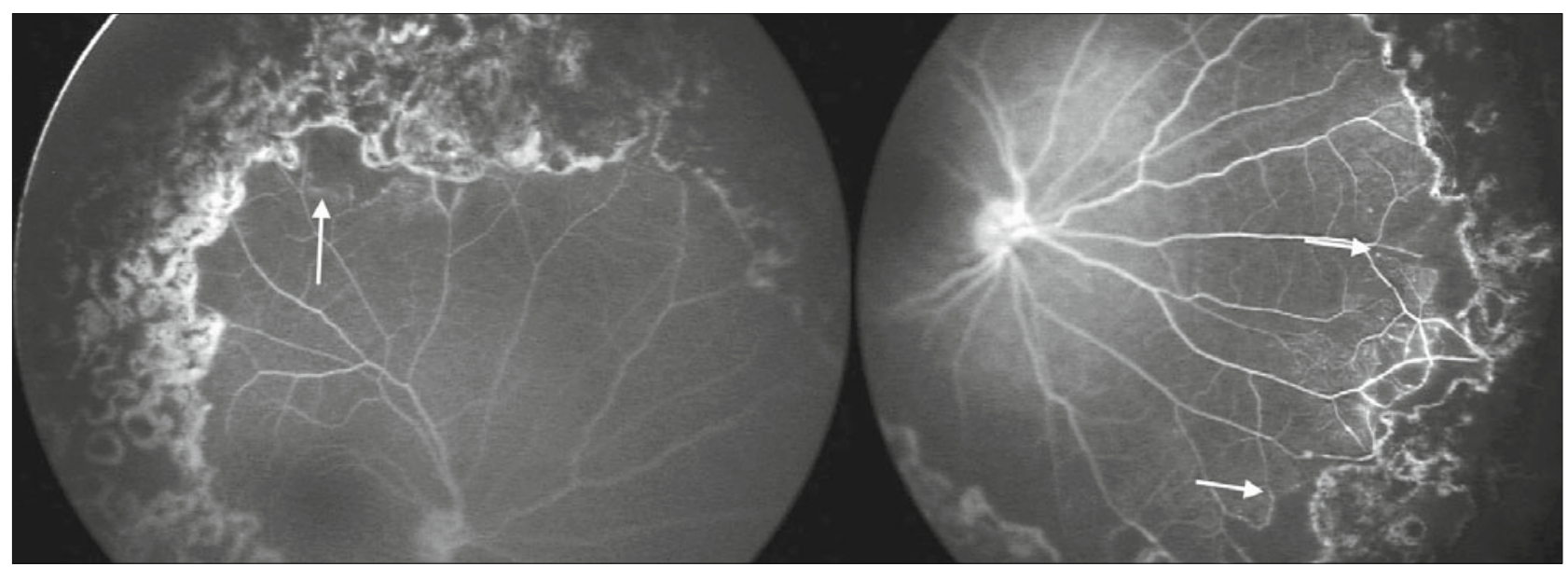

Fig. 5. FA of RE during control examination in February 2017 shows a small area of nonperfusion (arrows $\rightarrow$ ).

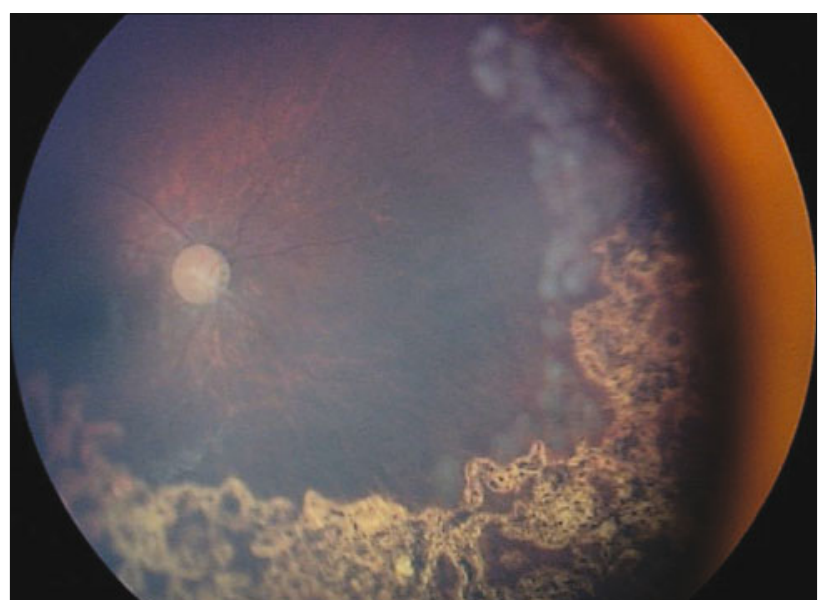

Fig. 6. Fundus of the RE after consecutive photocoagulation in March 2017.

mutation was found in cases of Norrie syndrome and severe forms of retinopathy of prematurity (ROP). Ophthalmological finding in Revesz syndrome can also mimic the cicatricial stage of ROP (5). Gupta et al (3) found a novel mutation in TINF2 gene, resulting in T284P TIN2 protein variant in Revesz syndrome. Ghadir et al found a heterozygous deletion TINF2 c 839delA in a 21-month old Hispanic male with Revesz syndrome and bilateral exudative retinopathy. They also describe a TINF2 mutation c865_866delinsAG, P289S in a patient with DC without ocular changes. In our case the mutation of TINF2 was found (variant c.865C > T;p.Pro289Ser) in a mosaic state with severe exudative retinopathy, which has not been described in accessible literature yet. In our case, FA and genetic tests helped establishing the correct diagnosis. Early diagnosis and identification of avascular areas and pathological retinal vascularisation allowed the early treatment, which is substantial for vision preservation.

\section{References}

1. Sharma A, Myers K, Ye Z, D'Orazio J. Dyskeratosis Congenita Caused by a Novel TERT Point Mutation in Siblings With Pancytopenia and Exudative Retinopathy. Pediatr Blood Cancer 2014; 61 (12): 2302-2304.

2. Thanos A, Todorich B, Hypes SM, Yonekawa Y, Thomas B, Randhawa S, Drenser KA, Trese MT. Retinal vascular tortuosity and exsudative retinopathy in a family with dyskeratosis congenita masquerading as familial exsudative vitreoretinopathy. Retin Cases Brief Rep 2017; 11 (Suppl 1): S187-S190.

3. Gupta MP, Talcott KE, Kim DY, Agarwal S, Mukai S. Retinal findings and a novel TINF2 mutation in Revesz syndrome: Clinical and molecular correlations with pediatric retinal vasculopathies. Ophthalmic Genet 2017; 38 (1): 51-60.

4. Savage SA. Dyskeratosis Congenita, GeneReviews. Seattle (WA): University of Washington, Seattle; 1993-2017.

5. McElnea EM, van der Spek N, Smith O, Fitzsimon S, Patel CK, O'Marcaigh A. Revesz syndrome masquerading as bilateral cicatricial retinopathy of prematurity. J AAPOS 2013; 17 (6): 634-636.

6. Sasa GS, Ribes-Zamora A, Nelson ND, Bertuch AA. Three novel truncating TINF2 mutations causing severe dyskeratosis congenita in early childhood. Clin Genet 2012; 81 (5): 10.1111/j.1399-0004.2011.01658.x. 